\title{
Clinical and Breed Characteristics of Idiopathic Head Tremor Syndrome in 291 Dogs: A Retrospective Study
}

\author{
Linda G. Shell, ${ }^{1}$ John Berezowski, ${ }^{2,3}$ Mark Rishniw, ${ }^{4}$ Belle M. Nibblett, ${ }^{1}$ and Patrick Kelly ${ }^{1}$ \\ ${ }^{1}$ Department of Clinical Sciences, Ross University School of Veterinary Medicine (RUSVM), \\ P.O. Box 334, Basseterre, Saint Kitts and Nevis \\ ${ }^{2}$ Department of Biomedical Sciences, Ross University School of Veterinary Medicine (RUSVM), \\ P.O. Box 334, Basseterre, Saint Kitts and Nevis \\ ${ }^{3}$ Veterinary Public Health Institute, Swiss Veterinary Faculty, University of Bern, Hochschulstrasse 4, 3012 Bern, Switzerland \\ ${ }^{4}$ Department of Clinical Studies, Cornell University, 602 Tower Road, Ithaca, NY 14853, USA \\ Correspondence should be addressed to Linda G. Shell; 1shell@rossvet.edu.kn
}

Received 7 January 2015; Accepted 17 April 2015

Academic Editor: Pedro J. Ginel

Copyright (c) 2015 Linda G. Shell et al. This is an open access article distributed under the Creative Commons Attribution License, which permits unrestricted use, distribution, and reproduction in any medium, provided the original work is properly cited.

\begin{abstract}
Objective. To establish signalment and phenomenology of canine idiopathic head tremor syndrome (IHTS), an episodic head movement disorder of undetermined pathogenesis. Design. Retrospective case series. Animals. 291 dogs with IHTS diagnosed between 1999 and 2013. Procedures. Clinical information was obtained from an online community of veterinary information aggregation and exchange (Veterinary Information Network, 777 W Covell Boulevard, Davis, CA 95616) and conducted with their approval. Information on breed, sex, age of onset, tremor description, mentation during the event, effect of distractions and drugs, diagnostics, presence of other problems, and outcome was analyzed. Results. IHTS was found in 24 pure breeds. Bulldogs, Labrador Retrievers, Boxers, and Doberman Pinschers comprised 69\%; mixed breeds comprised 17\%. Average onset age was 29 months (range: 3 months to 12 years). First episode occurred before 48 months of age in $88 \%$. Vertical (35\%), horizontal (50\%), and rotational (15\%) movements were documented. Possible trigger events were found in $21 \%$. Mentation was normal in $93 \%$. Distractions abated the tremor in $87 \%$. Most dogs did not respond to antiepileptic drugs. Conclusions and Clinical Relevance. This retrospective study documents IHTS in many breeds including Labrador Retrievers, Boxers, and mixed breeds.
\end{abstract}

\section{Introduction}

Canine idiopathic head tremor syndrome (IHTS), also sometimes referred to as episodic rapid repetitive myoclonus, is generally regarded as a benign condition manifesting as episodic uncontrolled head tremors that start and stop spontaneously. These head tremors have been reported to occur in "vertical" ("yes") or "horizontal" ("no") directions. Affected dogs appear alert during the tremors [1]. The diagnosis has been based on signalment, history, and characteristic head tremors as described above. Recent reports in the literature have characterized IHTS phenomenology in Doberman Pinschers [2] and English Bulldogs [3]. Although the cause of IHTS is unknown, affected Doberman Pinschers were traced to a common sire suggesting the condition may be inherited [1].
The veterinary literature, including neurology textbooks, does not contain significant information about IHTS for the practicing veterinarian. Affected dogs may initially be considered to have seizure activity, since this is the closest condition that might explain the signs. Thus, treatment with antiepileptic drugs (AEDs) may be attempted. Noting numerous cases of IHTS described by members of an online veterinary database (Veterinary Information Network, $777 \mathrm{~W}$ Covell Boulevard, Davis, CA 95616; this study was conducted with approval from Veterinary Information Network and its members), we sought to gain more information about IHTS by analyzing data from these cases. Specifically, we sought to document affected breeds, detail the character (duration and frequency) and direction of the head movement, investigate effects of stressful events and treatments on IHTS episodes, and determine whether or not the life span of affected dogs was affected. 


\section{Materials and Methods}

Data on IHTS cases was obtained in two surveys.

2.1. Survey 1. Clinical information was reviewed from veterinarians who participate in an online community of veterinary information aggregation and exchange (Veterinary Information Network, 777 W Covell Boulevard, Davis, CA 95616: this study was conducted with approval from Veterinary Information Network and its members). Archived cases were searched for by key words: head tremor syndrome, head bob, head bobble, head bobbing, head bobbers, bobble head, bobble doll, shakes head yes, and shakes head no; these are all descriptors that investigators (LGS and MR) had observed veterinarians use to describe clinical signs of IHTS. Cases with at least one key word were evaluated by two investigators (LGS and BMN or PK) to confirm that the following inclusion criteria were met: characteristic description of head tremors associated with IHTS (up and down motions or side to side motions), absence of limb or truncal tremors and other signs of cerebellar origin, short duration (seconds to minutes) of head tremors, intermittent occurrence of head tremors, and lack of other neurological signs. Inclusion was further supported by the opinion of one or more veterinary neurologists who agreed that the description of signs suggested a diagnosis of IHTS.

From the written data and from the available videos of cases, we created a database containing details of age, breed, sex, age of onset, tremor description and direction, duration (in seconds/minutes), frequency and timing of events, mentation during the event, effect of distraction and types of distractors, and diagnostics performed.

2.2. Survey 2. An online survey of veterinarians identified additional cases of IHTS from which data was collected and reviewed (LGS and MR). In the survey, veterinarians were given a written description of IHTS, viewed a video of IHTS in a dog, and were asked to provide information (age of onset of tremor, breed, sex, tremor description including frequency, direction, etc.) from their medical charts if they had managed a case that clinically fit the description of IHTS. In addition to the information collected in survey one, additional information was obtained on body position during tremors (standing, sternal, and lateral), presence of behavioral problems, other diseases and seizures, history of littermates with signs, drugs administered prior to or during the tremor, and outcome.

2.3. Statistical Analysis. Wilcoxon Rank Sum Tests, Fischer's Exact Tests, and Chi Squared tests were used to compare groups. All analyses were performed using standard statistical software ( $\mathrm{R}$ : a language and environment for statistical computing: Version i386.3.1.0. R Core Team (2014); R Foundation for Statistical Computing, Vienna, Austria; URL http://www.R-project.org/). A value of $P<0.05$ was considered significant.
TABLE 1: Breed distribution of 291 dogs with IHTS.

\begin{tabular}{lc}
\hline Breed & Percentage (number) \\
\hline Bulldog & $37 \%(107)$ \\
Mixed & $16 \%(48)$ \\
Boxer & $13 \%(37)$ \\
Labrador Retriever & $11 \%(33)$ \\
Doberman Pinscher & $8 \%(24)$ \\
Staffordshire Terrier & $3 \%(9)$ \\
Basset Hound & $1 \%(3)$ \\
Boston Terrier & $1 \%(3)$ \\
Golden Retriever & $1 \%(3)$ \\
Greyhound & $1 \%(3)$ \\
Parson Russell Terrier & $1 \%(3)$ \\
Beagle & $0.6 \%(2)$ \\
Great Dane & $0.6 \%(2)$ \\
Miniature Schnauzer & $0.6 \%(2)$ \\
Vizsla & $0.6 \%(2)$ \\
Australian Shepherd & $0.3 \%(1)$ \\
Border Collie & $0.3 \%(1)$ \\
English Springer Spaniel & $0.3 \%(1)$ \\
German Shorthaired Pointer & $0.3 \%(1)$ \\
Papillon & $0.3 \%(1)$ \\
Pembroke Welsh Corgi & $0.3 \%(1)$ \\
Redbone Coonhound & $0.3 \%(1)$ \\
Rhodesian Ridgeback & $0.3 \%(1)$ \\
Shetland Sheepdog & $0.3 \%(1)$ \\
Standard Poodle & $0.3 \%(1)$ \\
\hline Total & $100 \%(291)$ \\
\hline
\end{tabular}

\section{Results}

3.1. Study Population. Of 197 potential cases of IHTS reported in the online database between January 1999 and April 2013, descriptive information and data were sufficient to meet the inclusion criteria for 137 cases and of these, 50 had accompanying videos which were reviewed and confirmed to be representative of IHTS. Of the 174 responses from the online survey, 154 responses also met the inclusion criteria and were included in the study. Data obtained in the two surveys were very similar and hence were combined for the analyses reported below (291 cases).

3.2. Breed Data. Table 1 presents the breed data. Most dogs with IHTS were purebreds (84\%; 243/291) with Bulldogs being the most commonly reported $(37 \%, 107 / 291)$ followed by mixed breeds (16\%, 48/291), Boxers (13\%, 37/291), Labrador Retrievers (11\%, 33/291), and Doberman Pinschers $(8 \%, 24 / 291)$. Videos 1,2 , and 3 show the head tremors in a miniature Schnauzer, a Labrador Retriever, and a Boxer, respectively.

3.3. Age of Onset. Age of onset was available for 280 cases and varied from 3 to 144 months. Mean and median ages were 29 and 25 months, respectively. The majority (88\%) of dogs had 
TABLE 2: Direction of head tremor based on breeds affected.

\begin{tabular}{lcccc}
\hline Breed & Horizontal & Vertical & Rotary & Total \\
\hline Bulldog & $41 \%(32)$ & $42 \%(33)$ & $17 \%(13)$ & 78 \\
Boxer & $59 \%(17)$ & $34 \%(10)$ & $7 \%(2)$ & 29 \\
Labrador Retriever & $44 \%(11)$ & $44 \%(11)$ & $12 \%(3)$ & 25 \\
Doberman Pinscher & $48 \%(10)$ & $33 \%(7)$ & $19 \%(4)$ & 21 \\
Other pure breeds & $49 \%(16)$ & $33 \%(11)$ & $18 \%(6)$ & 33 \\
Mixed & $67 \%(29)$ & $19 \%(8)$ & $14 \%(6)$ & 43 \\
\hline Total & $50 \%(115)$ & $35 \%(80)$ & $15 \%(34)$ & 229 \\
\hline
\end{tabular}

their first episode of IHTS before 48 months of age. Bulldogs had a statistically significantly earlier age of onset (average 24 months) compared to the averages for the other breeds (32 months $)(P=0.017)$.

3.4. Sex. Overall, fewer females $(41 \% ; 115 / 279)$ were documented with IHTS than males $(59 \% ; 164 / 279)$. The percent of females varied by breed from a low of $27 \%$ females among Doberman Pinschers to a high of $51 \%$ in Bulldogs $(P=$ 0.158).

\subsection{Description of Head Tremor}

3.5.1. Direction of Head Tremor. Horizontal head motions ("no") occurred in half the dogs (115/229). Rotational motions were the least common, found only in $15 \%$ of the dogs (Table 2). There were no significant differences in the horizontal and vertical directions between the five most commonly affected breeds: Bulldogs, mixed breeds, Boxers, Labradors, and Dobermans $(P=0.306)$. Video 1 shows more of a rotational type of tremor in a miniature Schnauzer See Video 1 in Supplementary Materials available online at http://dx.doi.org/10.1155/2015/165463. Video 2 shows vertical head tremors in a Boxer and video 3 shows horizontal tremors in a Labrador Retriever.

3.5.2. Mentation. Ninety-three percent (246/264) of cases were considered to be alert or responsive during the episode. Only a few veterinarians described their patients' mentation as confused or disoriented (12), anxious (3), or lethargic (3).

3.5.3. Duration of Tremor. In the majority of affected dogs $(82 \% ; 202 / 245)$ the tremors lasted less than 5 minutes (Table 3) with only 15\% (37/245) lasting between 5 minutes and 1 hour. Three dogs were reported to have tremors occurring continuously over $12-48$ hours.

3.5.4. Frequency of Occurrence. Frequency data is presented in Table 4 . The most commonly reported occurrences were multiple times/day (26\%; 65/246) and every few days $(25 \%$; $61 / 246)$. However $22 \%$ (55/246) of the cases occurred infrequently based on combined results for the categories of "sporadically", "quarterly," and "less frequently than 3-4 months."

3.5.5. Body Position and Relationship to Rest. Affected dogs could be found in standing (41 cases), sitting (34 cases), or sternal ( 40 cases) positions at the onset of the tremor. Tremors
TABLE 3: Duration of head tremors.

\begin{tabular}{lcc}
\hline Time & Percent (number) & Cumulative \% \\
\hline 0 to 0.5 min & $21 \%(52)$ & 21 \\
0.5 to 1.0 min & $20 \%(48)$ & 41 \\
$1-2 \mathrm{~min}$ & $18 \%(43)$ & 58 \\
$2-5 \mathrm{~min}$ & $24 \%(59)$ & 82 \\
$5-60 \mathrm{~min}$ & $15 \%(37)$ & 98 \\
$1-2 \mathrm{hr}$ & $1 \%(3)$ & 99 \\
$>2 \mathrm{hr}$ & $1 \%(3)$ & 100 \\
\hline Total & $100 \%(245)$ & \\
\hline
\end{tabular}

TABLE 4: Frequency of occurrence of head tremors.

\begin{tabular}{lc}
\hline Frequency category & Percent (number) \\
\hline Continuously over 12-24 hours & $1 \%(3)$ \\
Multiple times/day & $26 \%(65)$ \\
Once a day & $9 \%(21)$ \\
Every few days & $25 \%(61)$ \\
Weekly & $7 \%(18)$ \\
Monthly & $9 \%(23)$ \\
Quarterly & $4 \%(9)$ \\
Less frequently than every 3-4 months & $10 \%(24)$ \\
Sporadically & $9 \%(22)$ \\
\hline Total & $100 \%(246)$ \\
\hline
\end{tabular}

did occur in lateral recumbency (5) but less commonly. Additional unprompted information indicated that head tremors were observed while the dog was resting, sleeping or waking from sleep in 66 cases.

3.5.6. Effect of Distractions. In $87 \%(142 / 163)$ of dogs, distractions caused the tremor to disappear for an undisclosed time. Distractors included voluntary turning of the head to one side or the other, calling the dog by its name, offering it food, making a sound so that the dog turned its head, and asking the dog to perform a task. More than one distractor was frequently reported as being helpful.

3.5.7. Time of Day That Tremor Was Observed. Most veterinarians $(76 \%)$ did not have information about the time of day the tremors occurred (220/291 cases). For 71 dogs, a time of day was known: $58 \%$ (41/71) had tremors occurring in the evening/night hours, 34\% (24/71) in the daytime hours, and $8 \%(6 / 71)$ during both day and night hours.

\subsection{Predisposing Factors}

3.6.1. Concurrent Medications. Forty-four percent (67/154) of affected dogs were receiving medications at the time of onset of IHTS. For the majority (85\%; 57/67), these medications were heartworm and flea control products; the rest of medications were prednisolone, diphenhydramine, tramadol, antibiotics, fludrocortisone, oral cyclosporine, and various eye medications. 
3.6.2. Concurrent Illness, Trauma, or Disease. Concurrent illness, trauma, or stressful events were noted in $21 \%$ of patients (33/154). Traumatic events (hit by car, dog fight), surgeries (castration, skin tumor removal, and cherry eye repair), and medical conditions (allergies; upper or lower respiratory infection; gastrointestinal, skin, or urinary tract infection; heart failure; demodicosis; heartworm disease; bee sting; papilloma; and immune mediated hemolytic anemia) had been diagnosed within a week (18 cases) or a month (6 cases) of the IHTS onset. Nine cases experienced a stressful event such as moving or a change in household members, travel, kennel confinement, or shock collar use. Specific triggers (gastrointestinal upset, shock collar training, and thunderstorm phobia) for repeated IHTS signs were identified in 3 cases.

3.6.3. Concurrent Behavioral Issues. History of behavioral problems was positive in $13 \%(20 / 153)$ of cases either prior to (10 cases), concurrently with (3 cases), or after (7 cases) the onset of IHTS. Behavioral problems included fear or aggression (8), thunderstorm phobia (4), separation anxiety (3), and unspecified problems (5).

3.6.4. Family History. Familial history was unknown for $89 \%$ $(137 / 154)$ of cases, reported positive for IHTS in 4/154 (2.6\%), and reported negative for IHTS in 13 . Of the 4 cases with familial information, the known affected dog had littermates (2) or unspecified family members (2) with IHTS.

\subsection{Outcome}

3.7.1. Treatment. Treatment was documented for $43 / 254$ (17\%) dogs. A variety of medications were used including bromide (5), phenobarbital (20), diazepam (12), and calcium carbonate (3). Less commonly used treatments included various antibiotics, corticosteroids, supplements (fish oil, antioxidants), clonazepam, and diphenhydramine. Veterinarians reported possible positive responses for oral maintenance phenobarbital (5/20), diazepam (3/12), calcium carbonate $(1 / 3)$, and fish oil (1/1).

3.7.2. Neurological Diagnostics. Results of cerebrospinal fluid analysis and advanced brain imaging were normal in 8 of 15 cases evaluated and unknown for 7 cases.

3.7.3. Development of Other Neurological Disorders. Other neurological disorders did not develop in the majority $(95 \%$; 146/154) of dogs after the onset of IHTS. The neurological signs or disorders that developed in 8 dogs (5\%) were diverse and were comprised of seizures (3) and one case of each of the following metronidazole-induced ataxia that resolved when medication was discontinued, degenerative myelopathy, idiopathic Horner's syndrome, cervical spondylopathy, and episodic collapse. Of the 3 cases that developed seizure activity, one developed it 9 years after IHTS was recognized; brain neoplasia was suspected but not proven. Another case developed seizures 2 months after onset of IHTS and subsequent results of spinal fluid analysis and brain magnetic resonance imaging studies were normal.

3.7.4. Long Term Outcome. Of the 135 dogs for which long term data was available, $91 \%$ were still alive; the remaining $9 \%$ had died or been euthanized for reasons unrelated to IHTS. All or most of the clinical signs of IHTS had resolved in $67 \%$ (90/135) while $24 \%(33 / 135)$ continued to have intermittent clinical signs.

\section{Discussion}

Currently the diagnosis of IHTS is a clinical one based on signalment (breed disposition), history (no exposure to toxins, intermittent nature of signs, etc.), lack of concurrent neurological and physical signs that might explain the tremors, and presence of characteristic head tremors occurring sporadically and of short duration. No diagnostic tests have been identified to date that allow a more specific diagnosis of IHTS. Likewise, no other diseases have been described to produce the same signs as IHTS. Only a few IHTS cases have been documented to have advanced diagnostics (spinal fluid analysis, brain scans) and in those no abnormalities were reported [2,3]. Therefore, the diagnosis of IHTS in this study was based on inclusion criteria that fit with IHTS as has been defined in other published studies [2, 3]. The authors acknowledge the inherent weakness of relying upon case descriptions and survey data but the clinical signs and historical data were sufficiently characteristic enough to hypothesize a diagnosis of IHTS and to eliminate cases that were not IHTS.

While previous studies centered on Bulldogs [2] and Doberman Pinschers [3], with an anecdotal report that Bulldog and Boxer breeds were commonly affected [1], we found IHTS in 24 pure breeds and in a substantial number of mixed breed dogs $(17 \%)$. Although there are no data on relative breed prevalence in the US, it did seem that certain breeds appeared overrepresented, mainly, Bulldogs (37\%), Boxers (13\%), Labrador Retrievers (11\%), and Doberman Pinschers $(8 \%)$. Our scant familial information and the propensity for certain breeds to be affected suggest a possible hereditary basis to the condition which is consistent with the report that affected Doberman Pinschers could be traced to a common sire [1].

Most dogs in our study had onset of signs at a mature age with $88 \%$ occurring before 4 years of age. This onset agrees with prior reports that suggested onset age was usually between 6 months and 3 years [1-3]. However it is important to note that age of onset varied from as early as 3 months of age to as old as 12 years.

The previously described phenomenology of IHTS in Bulldogs [3] and Doberman Pinschers [2] is generally similar to our findings across 24 breeds and mixed breed dogs. Generally, IHTS presents as head tremors in a vertical, horizontal, or rotary direction, usually in mature dogs under 4 years of age. Episodes are spontaneous in onset and sporadic in number and occurrence. Duration is variable from seconds to several hours but most episodes do not last longer than 
5 minutes. Tremors can occur while the dog is standing, resting, or sleeping. Most dogs are alert during an episode but some are anxious or lethargic. No drug therapy was identified that influenced the condition.

A number of new phenomenological findings emerged in our study. First, we documented the presence of a rotational head tremor in $15 \%$ of cases. This might be similar to the 9.2\% of affected Dobermans that Wolf et al. [2] described with motions in both vertical and horizontal directions, which could be construed as rotational [2]. The significance of direction of head tremors is unknown. Second, getting the dog's attention so that it moved its head seemed to make the tremor disappear or cease momentarily in $87 \%$ of our cases. Most of the distractors reported in this study as well as those in Wolf et al.'s [2] study would result in mental stimulation or a change in neck position. Why the head tremor would cease with a change in neck position is unknown but warrants further investigation into one proposed theory that IHTS involves the stretch reflex mechanism [4]. Finally, although the data were limited, there was more of a trend toward evening or nighttime occurrence. However, this could be explained by the time of day that most owners are home and interacting with or observing their pet.

The relationship of the head tremor with body/neck posture, rest, the sleep/wake cycle, and neck movement needs further investigation. In the Bulldog study, IHTS episodes occurred predominately at rest [3]. Likewise, many of the Doberman Pinschers were reported to be reclining or dozing when the IHTS started [2]. Dogs in our study were reported to manifest IHTS while resting or being in stationary positions (sitting, standing, or being in sternal or lateral recumbency). The condition did not appear to be initiated by movement.

This study confirmed that the majority of cases do not have any other neurological or behavioral problems. However, stressful events or concurrent illness or trauma, occurring predominately within a week of the tremor onset, was noted in about one-fifth of our cases. In prior studies, stressful events were associated with IHTS in $46.7 \%$ of Dobermans and in $7 \%$ of Bulldogs $[2,3]$. Thus underlying diseases and environmental stressors should be investigated in any dog presenting with signs of IHTS.

Outcome obtained from 135 cases indicated that the majority of affected dogs were still alive (91\%) and that none had died or been euthanized because of IHTS. Thus IHTS is not a life-threatening disease. Furthermore the intermittent head tremors eventually disappeared in the majority of affected dogs (67\%) although the survey did not gather data on the time it took for signs to resolve. One might assume that if underlying diseases and environmental stressors are addressed, clinical signs may resolve. Intermittent signs were reported, however, to occur in some dogs.

Focal seizure activity has been mentioned as a possible cause of IHTS [2] but currently there is little to no data to support that possibility. Other authors [4] have noted that AEDs do not appear effective in treating this disorder; that appeared to be the case for the few dogs that were medicated with maintenance AEDs in our study. We acknowledge, however, that, given the sporadic nature and short duration of clinical signs, it would be difficult to access AED efficacy; likewise, for the same reasons, it would be difficult to justify using AEDs in these patients unless more obvious generalized seizure activity was documented.

Unfortunately, tremors have many possible causes. In humans, tremors are the most common type of movement disorders [5-7] but tremors can also occur with stereotypies, tics, and psychogenic disorders $[8,9]$. Tremors can also be a part of other movement disorders such as essential tremor syndrome, cervical dystonia [10], and paroxysmal dyskinesias [11]. However, tremors associated with these movement disorders are not paroxysmal, nor is the head a commonly affected area. Thus at the present time, IHTS does not appear to be similar to known movement disorders in humans.

In summary, our study shows that IHTS can be found in a wide range of dog breeds. The data we collected more clearly defines the clinical presentation and signs. IHTS is a benign condition that frequently resolves spontaneously and is nonresponsive to treatment with a wide range of conventional drugs. The condition is of concern to dog owners, however, and further studies appear warranted to establish its pathogenesis.

\section{Disclosure}

This paper is presented partially in abstract form at the 26th Annual ESVN-ECVN Symposium, Paris, France, September 2013.

\section{Conflict of Interests}

The authors declare that there is no conflict of interests regarding the publication of this paper.

\section{Acknowledgments}

The authors thank Veterinary Information Network and the many veterinarian members for their contributions to this study and RUSVM veterinary medicine students who assisted in verifying data. It is supported in part by RUSVM, Center for Integrative Mammalian Research.

\section{References}

[1] A. De Lahunta, E. N. Glass, and M. Kent, "Classifying involuntary muscle contractions," Compendium on Continuing Education for the Practicing Veterinarian, vol. 28, no. 7, pp. 516-529, 2006.

[2] M. Wolf, A. Bruehschwein, C. Sauter-Louis, A. C. Sewell, and A. Fischer, "An inherited episodic head tremor syndrome in Doberman pinscher dogs," Movement Disorders, vol. 26, no. 13, pp. 2381-2386, 2011.

[3] J. Guevar, S. DeDecker, L. M. L. Van Ham, A. Fischer, and H. A. Volk, "Idiopathic head tremor in English bulldogs," Movement Disorders, vol. 29, no. 2, pp. 191-194, 2014.

[4] A. Delahunta and E. Glass, "Upper motor neuron," in Veterinary Neuroanatomy and Clinical Neurology, pp. 192-220, Saunders, St. Louis, Mo, USA, 3rd edition, 2009. 
[5] G. Grimaldi and M. Manto, "Definition of tremor," in Mechanisms and Emerging Therapies in Tremor Disorders, G. Grimaldi and M. Manto, Eds., pp. 3-10, 2012.

[6] P. Crawford and E. E. Zimmerman, "Differentiation and diagnosis of tremor," American Family Physician, vol. 83, no. 6, pp. 697-702, 2011.

[7] S. Smaga, “Tremor," American Family Physician, vol. 68, no. 8, pp. 1545-1553, 2003.

[8] E. F. Augustine and J. W. Mink, "Tic disorders and stereotypies," in Oxford Textbook of Movement Disorders, D. Burn, Ed., pp. 247-254, 2013.

[9] S. G. Reich, "Psychogenic movement disorders," Seminars in Neurology, vol. 26, no. 3, pp. 289-296, 2006.

[10] C. Godeiro-Junior, A. C. Felicio, P. C. Aguiar, V. Borges, S. M. A. Silva, and H. B. Ferraz, "Head tremor in patients with cervical dystonia: different outcome?" Arquivos de Neuro-Psiquiatria, vol. 66, no. 4, pp. 805-808, 2008.

[11] K. P. Bhatia, "Familial (idiopathic) paroxysmal dyskinesias: an update," Seminars in Neurology, vol. 21, no. 1, pp. 69-74, 2001. 

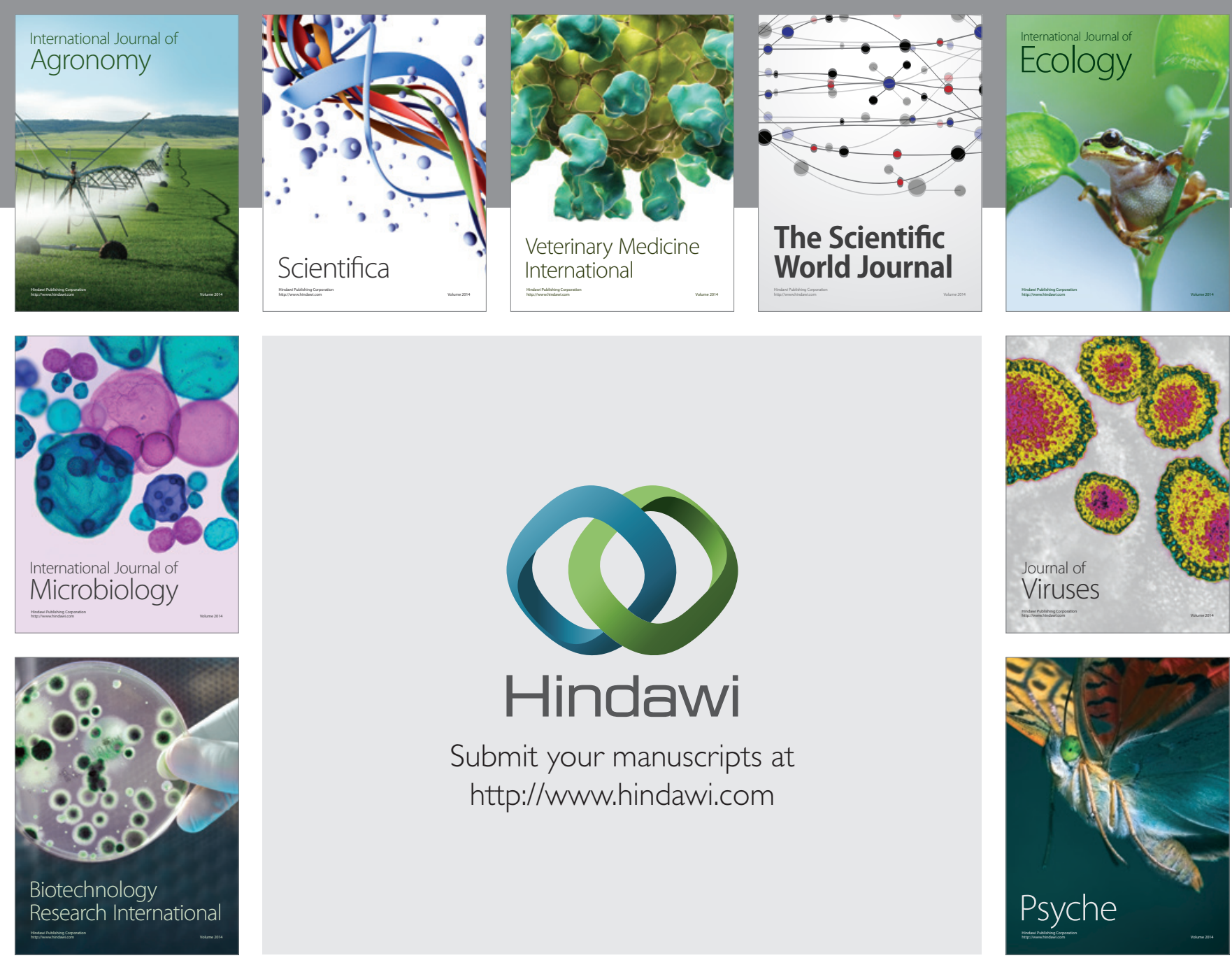

Submit your manuscripts at http://www.hindawi.com
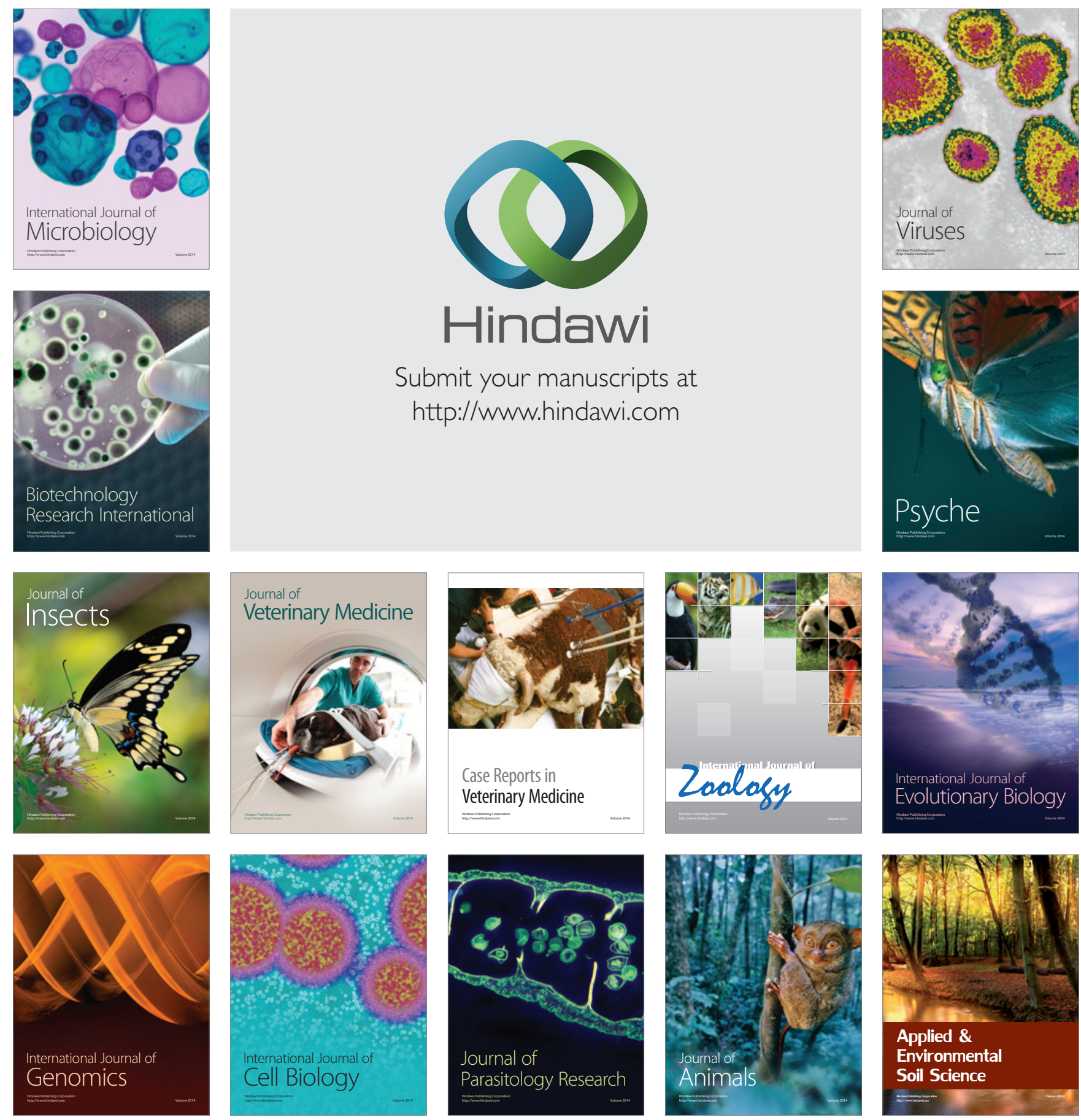